\title{
Voyager pour une radiothérapie du cancer : la satisfaction des patients
}

\author{
Par Margaret I. Fitch, Ross E. Gray, Tom McGowan, \\ Ian Brunskill, Shawn Steggles, Scott Sellick, \\ Andrea Bezjak et Donna McLeese
}

\section{Résumé}

Cette étude a pour but de décrire la satisfaction des personnes atteintes du cancer envers les soins qu'elles ont reçus lorsqu'elles ont dî s'éloigner subitement de leur région pour bénéficier d'un traitement. L'Ontario a lancé un programme de réacheminement pour les personnes atteintes du cancer qui avaient besoin d'une radiothérapie, lorsque les listes d'attente dans le sud de la province sont devenues trop longues. Des patients ont ainsi dû se rendre aux États-Unis ou dans le nord de l'Ontario pour se faire soigner. À l'issue de leur traitement, un questionnaire normalisé de 25 questions dont les réponses étaient sous la forme d'une échelle de type Likert en cinq points a été envoyé par la poste à tous les patients ayant participé au programme de réacheminement. Les questions portaient sur les expériences des patients avant leur départ, les dispositions de voyage et le séjour dans les établissements d'accueil associés au centre de cancérologie, à l'extérieur de leur région. Au total, 466 patients $(55,8 \%)$ ont retourné le questionnaire. Dans l'ensemble, ils se montraient satisfaits des soins. Cependant, des améliorations leur ont paru souhaitables dans un certain nombre de domaines, tels que l'accès à un soutien avant de s'éloigner de leur région, l'accès à l'information sur les services en soins de soutien lors de leur séjour à l'extérieur, et la prise en compte de leurs besoins personnels dans l'organisation du voyage. Pour les personnes atteintes du cancer qui doivent voyager en vue d'un traitement, la prestation d'information et le soutien sont d'une grande importance.

L'impact sur un individu d'un diagnostic de cancer et du traitement qui en découle n'est pas seulement physique. L'impact ressenti peut être émotionnel, social, psychologique, spirituel ou pratique (CarrollJohnson, Gorman et Bush, 1998). Les bouleversements qui résultent de cet impact peuvent susciter d'innombrables défis, et affronter ces défis peut s'avérer bien difficile pour l'individu et ses proches (Abbey-Livingstone and Associates, 1990; Société canadienne du cancer, 1992).

La nature de la radiothérapie du cancer exige que les patients se rendent quotidiennement au centre de traitement et ce, pendant 4 à 5 semaines. Les patients qui habitent trop loin du centre de traitement et qui n'ont pas la possibilité de conduire tous les jours, peuvent être forcés de s'absenter de leur région durant cette période et à séjourner dans des locaux d'hébergement situés à proximité du centre de cancérologie. Le fait de ne pas être chez soi entre les séances de traitement risque d'ajouter à la détresse qu'un individu peut éprouver, à plus forte raison lorsque le voyage est inattendu.

En 1999, pour des raisons diverses, plusieurs centres de cancérologie du sud de l'Ontario furent dans l'incapacité de prodiguer en temps opportun une radiothérapie à tous les patients qui exigeaient un tel traitement. Des patients, qui auraient pu normalement rester chez eux entre les séances de traitement, ont été réorientés vers des centres de cancérologie du Nord de l'Ontario ou des États-Unis. Un programme de réacheminement officiel a été mis en oeuvre pour réorienter certains patients vers une destination qu'ils avaient choisie dans une liste arrêtée au préalable, et leur apporter l'aide nécessaire à leur voyage et à leur hébergement. Ce programme prévoyait également le remboursement des frais occasionnés par le réacheminement vers un autre centre.

Il existe peu d'études systématiques des points de vue des patients sur de tels déplacements. Given, Given et Harlan (1994), décrivent l'isolement géographique comme l'un des facteurs influençant l'accès aux soins anticancéreux en milieu rural dans le Michigan. Là où les services de soins anticancéreux sont déficients, la population rurale du Michigan est souvent exclue du traitement adjuvant de pointe qui nécessite des visites quotidiennes ou hebdomadaires dans les centres de traitement. Les habitants ruraux d'Australie sont aussi isolés géographiquement et confrontés aux problèmes inhérents à la nécessité de parcourir de longues distances, surtout pour les soins spécialisés (McGrath et al., 1999a). Lorsque les personnes atteintes du cancer doivent voyager pour recevoir un traitement, elles se disent préoccupées par leurs responsabilités familiales et professionnelles, s'inquiètent de la manière dont leur famille s'en sortira en leur absence, ainsi que des charges financières (Davis et Girgis, 1998; McGrath et al., 1999b).

À ce jour, une seule étude canadienne s'est concentrée sur la difficulté de voyager pour des personnes atteintes du cancer qui suivent une radiothérapie (Fitch et al., 2003). Dans cette étude, trois groupes de patients atteints de cancer du sein et de la prostate ont été interrogés : a) des patients ayant été subitement réorientés vers le nord de l'Ontario ou les États-Unis pour y suivre leur traitement, b) des patients restés dans leur région durant le traitement, et c) des patients domiciliés dans le nord de l'Ontario, qui parcouraient normalement

Margaret I. Fitch, PhD, Unité de recherche psychosociale et comportementale, Centre régional de cancérologie de Toronto Sunnybrook, Coordinatrice, Soins de soutien, Action Cancer Ontario.

Ross E. Gray, PhD, Unité de recherche psychosociale et comportementale, Centre régional de cancérologie de Toronto Sunnybrook.

Tom McGowan, MD, FRCPC, Action Cancer Ontario.

Ian Brunskill, MBA, CHE, Action Cancer Ontario.

Shawn Steggles, PhD, Centre régional de cancérologie du NordEst de l'Ontario.

Scott Sellick, PhD, Centre régional de cancérologie du Nord-Est de l'Ontario.

Andrea Bezjak, MD, FRCPC, Hôpital Princess Margaret.

Donna McLeese, RT(T), Action Cancer Ontario.

At time of study 
des distances comparables à celles du premier groupe. Les conclusions ont révélé que les personnes atteintes du cancer éprouvent des difficultés à voyager pour suivre un traitement, que ce déplacement soit prévu ou non. Pour celles qui séjournent à l'hôtel ou dans une résidence à proximité du centre de cancérologie, les interactions avec d'autres malades sont d'un grand secours face à cette épreuve.

Le programme de réacheminement des patients de l'Ontario a été lancé à titre de programme provincial, à un moment où les délais d'attente des radiothérapies étaient jugés inacceptables par les radio-oncologues de plusieurs centres de cancérologie du sud de la province. Des arrangements ont été passés avec deux centres canadiens (Sudbury et Thunder Bay) et cinq centres des États-Unis (Buffalo - 2 établissements, Cleveland, Rochester et Detroit) dans le but de proposer aux patients d'y suivre leur radiothérapie. Un bureau central a été créé pour assurer la coordination des réacheminements. Au gré des entretiens avec leur médecin ou leur chirurgien, les personnes atteintes de cancer du sein et de cancer de la prostate se sont vues proposer l'option d'être réorientées pour leur traitement vers un centre de cancérologie en dehors de leur région, ou celle d'attendre un traitement dans un centre de lutte contre le cancer le plus proche de leur lieu de résidence. Le personnel du bureau central de réacheminement s'occupait ensuite du transfert des patients qui avaient fait le choix d'être réorientés vers un autre centre de soins, de leur séjour dans un hôtel désigné à cet effet et du remboursement des frais de voyage, de nourriture et de logement. Avant d'entreprendre leur voyage, la plupart des patients n'avaient pas eu affaire à un radio-oncologue ni au personnel de leur centre de cancérologie local. Les patients qui choisissaient de rester chez eux et d'être traités dans le centre le plus proche étaient inscrits sur la liste d'attente du centre en question. Par la suite, ils ne recevaient aucun remboursement des dépenses liées à leur traitement.

Cette étude a été réalisée pour rendre compte de la satisfaction des patients vis-à-vis des soins qu'ils ont reçus dans le cadre du voyage inattendu effectué en vue de suivre leur radiothérapie en dehors de leur région. On escomptait en retirer des stratégies qui pourraient être mises en œuvre pour répondre aux besoins exprimés par les patients. Cet article présente les données recueillies au cours des douze mois suivant la mise en oeuvre du programme de réacheminement des patients de la province de l'Ontario. Il constitue une approche et un outil d'évaluation pouvant servir à d'autres programmes de lutte contre le cancer qui pourraient se trouver dans l'obligation d'envoyer des patients suivre une radiothérapie à l'extérieur de leur région.

\section{Méthodes}

Cette étude employait un sondage pour évaluer le degré de satisfaction des patients envers les soins qu'ils ont reçus dans le cadre de leur réacheminement pour une radiothérapie du cancer. L'autorisation déontologique a été obtenue du Comité d'éthique de la recherche de Sunnybrook and Women's College Health Sciences Centre.

L'échantillon de l'enquête regroupe toutes les personnes atteintes du cancer qui avaient été sélectionnées dans le cadre du programme de réacheminement pour suivre une radiothérapie dans un centre de cancérologie situé en dehors de leur région. Les critères d'éligibilité pour le programme de réacheminement s'établissaient comme suit : 1)diagnostic de cancer du sein ou de la prostate et 2) patient nécessitant une radiothérapie comme traitement de première intention, sauf en présence d'une des conditions suivantes : atteinte neurologique, maladie intercurrente, métastases à distance, canule ou sonde de trachéostomie, gastrostomie, jéjunostomie ou néphrostomie, besoins spéciaux en oxygène, thérapie anticancéreuse concurrente (c.-à-d. chimiothérapie), nécessité d'un traitement multidisciplinaire spécialisé, patient ne maîtrisant pas bien l'anglais, patient ayant la charge de jeunes enfants ou d'autres membres de la famille, et enfin, patient âgé de moins de 21 ans.

Les patients ont été sollicités par courrier à la fin de leur traitement, après avoir achevé leurs séances de radiothérapie au centre de cancérologie situé à l'extérieur de leur région. Des trousses, contenant une lettre explicative et une invitation à participer à l'étude, une copie du sondage sur la satisfaction, ainsi qu'une enveloppe-réponse timbrée, ont été envoyées à tous les patients qui avaient été réorientés. Les patients qui n'avaient pas retourné le questionnaire au bout d'un mois se sont vus adresser un avis de rappel par téléphone. Une seconde trousse a été envoyée à ceux qui avaient égaré la première et désiraient toujours prendre part au sondage.

$\mathrm{Au}$ total, 907 questionnaires ont été expédiés durant les 12 premiers mois du programme de réacheminement, et 466 ont été retournés (soit un taux de réponse de 55,8\%).

Enquête de satisfaction : L'enquête de satisfaction du réacheminement a été spécialement conçue pour cette étude. Le questionnaire a été formulé pour permettre aux patients d'exprimer leur satisfaction vis-à-vis d'événements ayant lieu avant ou pendant le traitement de radiothérapie. Les questions portaient sur les expériences suivantes : 1re partie) visites initiales au centre de cancérologie le plus proche de leur lieu de résidence (10 questions), 2e partie) préparatifs du voyage (3 questions), et $3 \mathrm{e}$ partie) visites au centre de cancérologie de réacheminement (12 questions). Les questions spécifiques concernaient l'accès à l'information, les modalités d'inscription, les dispositions de voyage, les facilités d'hébergement, la communication avec le personnel, l'accès aux services de soutien et la confiance dans les soins. Toutes les questions étaient des questions fermées et exigeaient des patients qu'ils indiquent leur accord ou leur désaccord sur une échelle de Likert en 5 points. Outre l'option « sans objet », les réponses possibles à toutes les questions étaient : «fortement en accord », « en accord », «neutre », « en désaccord » et « fortement en désaccord ». L'option « sans objet » a été ajoutée pour le cas où certains patients n'auraient pas effectué de visite auprès d'un centre de cancérologie local avant d'être réorientés vers un autre centre. Trois experts des soins aux patients en radiothérapie ont examiné les questions du sondage, afin d'en dégager la validité apparente.

\section{Analyse}

L'analyse des données de l'enquête de satisfaction est de nature descriptive. Les patients ont été regroupés selon des intervalles de trois mois, en fonction de la date à laquelle ils avaient voyagé pour leur radiothérapie. L'examen des données trimestrielles se justifiait par le fait que voyager durant les hivers canadiens peut susciter davantage de défis qu'à d'autres périodes de l'année. La fréquence des réponses à chacune des questions a été calculée pour chaque intervalle de trois mois (avril-juin $n=98$; juillet-septembre $n=121$; octobre-décembre $n=164$; janvier-mars $n=83$ ), ainsi que pour l'échantillon global $(\mathrm{N}=466)$. Une seconde analyse a été effectuée sur l'ensemble de l'échantillon pour déterminer si la satisfaction exprimée par les patients variait dans des proportions significatives en fonction du sexe et de la situation géographique du lieu de traitement (Canada/États-Unis). Le test du khi carré a été appliqué à chaque cas avec un seuil de signification établi à $\mathrm{p}=0,05$.

\section{Résultats \\ Échantillon}

Un total de 466 patients ont répondu à l'enquête de satisfaction. La moyenne d'âge des répondants était de 65,6 ans (écart type $=8,1$ ). Cinquante-huit pour cent $(\mathrm{n}=296)$ des répondants étaient des femmes. Soixante-douze pour cent $(n=364)$ avaient été réorientés vers les États-Unis pour une radiothérapie, le reste des patients s'étant déplacé vers le Nord de l'Ontario. 
Satisfaction envers les soins avant le déplacement

Les proportions de personnes interrogées qui ont indiqué leur satisfaction vis-à-vis de divers aspects des soins sont présentées dans le tableau $\mathrm{n}^{\circ} 1$, durant la période précédant leur déplacement. Dans l'ensemble, la majorité des patients ayant fréquenté un centre de cancérologie proche de leur lieu de résidence, avant de consentir à un réacheminement, ont indiqué qu'ils étaient satisfaits de ces soins. Les patients ont convenu que le personnel du centre de cancérologie local s'était montré courtois et plein d'égards (94\%) et qu'ils étaient parfaitement conscients que la décision de s'éloigner de chez eux pour leur traitement leur appartenait $(90 \%)$. Entre le moment du diagnostic et le début de la radiothérapie à l'extérieur, les patients estimaient qu'ils avaient un accès adéquat aux médecins et aux infirmières $(69 \%)$ et se sentaient soutenus par le personnel du centre de cancérologie local (70 \%). Environ trois quarts des patients $(73 \%)$ avaient confiance dans les soins de suivi

Tableau 1. Satisfaction des patients envers le Programme de réacheminement : questions portant sur le centre de traitement du cancer le plus proche du domicile du patient avant le voyage (échantillons trimestriels et globaux)

\begin{tabular}{|c|c|c|c|c|c|}
\hline Questions de la partie 1 & $\begin{array}{c}\text { Avr. -juin } 1999 \\
(\mathrm{n}=98)\end{array}$ & $\begin{array}{l}\text { Juil.-sept. } 1999 \\
\qquad(n=121)\end{array}$ & $\begin{array}{l}\text { Oct.-déc. } 1999 \\
(n=164)\end{array}$ & $\begin{array}{c}\text { Jan.-mars } 2000 \\
(\mathrm{n}=83)\end{array}$ & $\begin{array}{c}\text { Total } \\
(n=466)\end{array}$ \\
\hline & \multicolumn{5}{|c|}{$\begin{array}{c}\text { Proportion de répondants de chaque groupe } \\
\text { qui étaient d'accord avec l'énoncé }\end{array}$} \\
\hline $\begin{array}{l}\text { 1. Avant ma première visite au centre de } \\
\text { traitement du cancer le plus proche de } \\
\text { mon domicile, j'ai reçu une } \\
\text { information opportune et utile sur ce } \\
\text { à quoi je devais m'attendre. }\end{array}$ & $68 \%$ & $76 \%$ & $68 \%$ & $71 \%$ & $73 \%$ \\
\hline $\begin{array}{l}\text { 2. Le processus d'inscription au centre } \\
\text { de traitement du cancer le plus proche } \\
\text { de mon domicile était simple. }\end{array}$ & $90 \%$ & $88 \%$ & $86 \%$ & $81 \%$ & $87 \%$ \\
\hline $\begin{array}{l}\text { 3. Le personnel du centre de traitement } \\
\text { du cancer était courtois et respectueux. }\end{array}$ & $96 \%$ & $92 \%$ & $92 \%$ & $96 \%$ & $94 \%$ \\
\hline $\begin{array}{l}\text { 4. Lors de ma visite initiale, on m'a } \\
\text { donné une explication complète de } \\
\text { ma condition médicale et des } \\
\text { options de traitement. }\end{array}$ & $85 \%$ & $86 \%$ & $80 \%$ & $81 \%$ & $83 \%$ \\
\hline $\begin{array}{l}\text { 5. Il était clair pour moi que la décision } \\
\text { de voyager vers une autre ville pour } \\
\text { recevoir ma radiothérapie me revenait } \\
\text { à moi seul(e) et que j’avais le } \\
\text { droit de refuser. }\end{array}$ & $95 \%$ & $90 \%$ & $88 \%$ & $86 \%$ & $90 \%$ \\
\hline $\begin{array}{l}\text { 6. Au cours de mon rendez-vous initial, } \\
\text { j'ai eu l'impression que j'avais tout le } \\
\text { temps nécessaire pour discuter des } \\
\text { questions qui étaient importantes } \\
\text { à mes yeux. }\end{array}$ & $88 \%$ & $84 \%$ & $85 \%$ & $80 \%$ & $85 \%$ \\
\hline $\begin{array}{l}\text { 7. J'ai reçu tous les renseignements dont } \\
\text { j'avais besoin sur les démarches } \\
\text { nécessaires à l'obtention de ma } \\
\text { radiothérapie dans une autre ville. }\end{array}$ & $87 \%$ & $89 \%$ & $76 \%$ & $70 \%$ & $82 \%$ \\
\hline $\begin{array}{l}\text { 8. Durant la période qui a précédé mon } \\
\text { départ vers l'autre ville, j'avais } \\
\text { l'impression d'avoir accès aux } \\
\text { médecins et aux infirmières selon } \\
\text { mes besoins. }\end{array}$ & $64 \%$ & $75 \%$ & $66 \%$ & $73 \%$ & $69 \%$ \\
\hline $\begin{array}{l}\text { 9. Entre l'annonce du diagnostic et le } \\
\text { début de ma radiothérapie, j'avais } \\
\text { l'impression de recevoir un bon } \\
\text { soutien de la part des professionnels } \\
\text { de la santé du centre de traitement du } \\
\text { cancer le plus proche de chez moi. }\end{array}$ & $70 \%$ & $74 \%$ & $64 \%$ & $76 \%$ & $70 \%$ \\
\hline $\begin{array}{l}\text { 10. J'étais sûr(e) que l'équipe médicale } \\
\text { du centre de traitement du cancer le } \\
\text { plus près de chez moi me fournirait } \\
\text { des soins de suivi de qualité lors de } \\
\text { mon retour de la ville où j'avais } \\
\text { suivi ma radiothérapie. }\end{array}$ & $74 \%$ & $70 \%$ & $75 \%$ & $72 \%$ & $73 \%$ \\
\hline
\end{tabular}


que le personnel de ce centre de cancérologie leur dispenserait à leur retour $(73 \%)$.

En général, la proportion de patients satisfaits des soins au centre de cancérologie de leur région a peu varié tout au long de la période de douze mois. La baisse la plus significative (de 87 à $70 \%$ ) concernait la prestation d'information sur les démarches nécessaires à l'obtention d'une radiothérapie dans une autre ville. Bien que moins spectaculaires, d'autres baisses sont à signaler, notamment la proportion de patients satisfaits des modalités d'inscription au centre de cancérologie de leur région (de 90 à $81 \%$ ), la quantité de temps dont ils ont disposé pour discuter des questions importantes (de 88 à $80 \%$ ), et les explications fournies aux patients à propos de leur condition médicale et des options de traitement (de 85 à $81 \%$ ).

Satisfaction vis-à-vis de la coordination des dispositions de voyage

Les proportions de patients satisfaits de la coordination des dispositions de voyage vers un autre centre de cancérologie pour leur radiothérapie sont présentées dans le tableau n ${ }^{\circ} 2$. À terme, la proportion de patients satisfaits décroît sur les trois points concernant la coordination. La baisse la plus spectaculaire concerne 1'opportunité et l'efficacité de l'organisation des dispositions (de 90 à $68 \%$ ). En général, les trois quarts $(76 \%$ ) des répondants ont le sentiment que leurs besoins et ceux de leur famille ont été pris en compte lors de l'organisation des dispositions.

\section{Satisfaction envers les soins dispensés} au centre de cancérologie de destination

Les proportions de patients satisfaits des soins qu'ils ont reçus au centre de cancérologie de destination sont présentées dans le tableau $n^{\circ} 3$. Dans l'ensemble, les patients ont jugé favorablement de nombreux aspects des soins dont ils ont fait l'objet. Parmi ceux ayant suscité le moins d'enthousiasme, on remarque la charge financière imposée par le voyage $(56 \%)$, la prestation d'information sur les services de soins de soutien $(71 \%)$, et la fourniture d'informations utiles sur le centre de cancérologie de destination (79 \%) avant de s'y rendre pour le traitement. Les préoccupations à propos de la charge financière et des services en soins de soutien étaient manifestes tout au long de la période des douze mois, tandis que celles concernant les informations fournies sur le centre de cancérologie se faisaient plutôt sentir vers la fin de cette période.

\section{Satisfaction par sexe}

Les proportions de femmes et d'hommes interrogés indiquant qu'ils étaient satisfaits de leurs soins ont été comparées par l'analyse du Khi carré. Les quatre seuls points dénotant des différences significatives concernaient 1) le fait de bien comprendre que la décision de voyager était l'entière décision du patient, 2) le fait de recevoir l'information nécessaire aux dispositions du voyage, 3) la prise en compte des besoins du patient et de sa famille dans les dispositions du voyage, et 4) l'information fournie sur les services de soins de soutien. Les femmes étaient plus réservées que les hommes sur ces points (voir tableau $\mathrm{n}^{\circ} 3$ ).

\section{Satisfaction par contexte de traitement}

Les proportions de personnes interrogées satisfaites des soins dans les centres canadiens ont été comparées à celles des centres des ÉtatsUnis. Un seul point a révélé un écart significatif. Quatre-vingt-quatre pour cent des patients traités dans des centres canadiens étaient satisfaits de l'information fournie sur les services de soins de soutien par rapport à $66 \%$ dans les centres aux États-Unis (DDL $=1$, Chi $=8,64, \mathrm{P}=0,005)$.

\section{Discussion}

Cette étude a été entreprise pour vérifier la satisfaction des personnes atteintes du cancer envers les soins dont elles ont bénéficié, en suivant une radiothérapie dans un établissement à l'extérieur de leur région. Le fait d'accepter un réacheminement impliquait pour les patients un déplacement vers une autre ville pour la durée de leur radiothérapie. Ceux qui sont allés dans le Nord de l'Ontario ont voyagé en avion, approximativement $400 \mathrm{~km}$ jusqu'à Sudbury et 1400 km jusqu'à Thunder Bay. Ceux qui, depuis le Sud de l'Ontario, se sont rendus dans des établissements aux États-Unis, ont parcouru le plus souvent de 150 à $200 \mathrm{~km}$ en voiture ou en autocar.

La première limite de cette étude tient au taux de réponse de $55,7 \%$, malgré la relance téléphonique aux participants. Le taux de réponse a été cependant supérieur à celui de la plupart des enquêtes menées par courrier sans avis de rappel. Une autre limite, commune à tous les sondages expédiés par la poste, provient du fait que les gens qui prennent la peine de remplir et de renvoyer les formulaires sont probablement des individus intéressés par le sujet, à l'aise lorsqu'il s'agit de lire un questionnaire et d'y répondre. On peut regretter que des renseignements concernant le niveau d'instruction n'aient pas été réunis dans le cadre de cette étude.

Tableau 2. Satisfaction des patients envers le Programme de réacheminement : questions portant sur la coordination du voyage vers la ville où la radiothérapie a été suivie (échantillons trimestriels et globaux)

\begin{tabular}{|c|c|c|c|c|c|}
\hline Questions de la partie 2 & $\underset{(n=98)}{\text { Avr. -juin } 1999}$ & $\begin{array}{l}\text { Juil.-sept. } 1999 \\
\qquad(\mathrm{n}=121)\end{array}$ & $\begin{array}{l}\text { Oct.-déc. } 1999 \\
(n=164)\end{array}$ & $\begin{array}{c}\text { Jan.-mars } 2000 \\
(n=83)\end{array}$ & $\begin{array}{c}\text { Total } \\
(n=466)\end{array}$ \\
\hline & \multicolumn{5}{|c|}{$\begin{array}{c}\text { Proportion de répondants de chaque groupe } \\
\text { qui étaient d'accord avec l'énoncé }\end{array}$} \\
\hline $\begin{array}{l}\text { 11. Les dispositions de voyage afin que je } \\
\text { me rende dans une autre ville pour y } \\
\text { recevoir ma radiothérapie ont été } \\
\text { faites en temps opportun et de } \\
\text { manière efficace. }\end{array}$ & $90 \%$ & $90 \%$ & $75 \%$ & $68 \%$ & $81 \%$ \\
\hline $\begin{array}{l}\text { 12. La personne qui m'a aidé(e) à faire } \\
\text { les dispositions de voyage était } \\
\text { courtoise et respectueuse. }\end{array}$ & $95 \%$ & $97 \%$ & $91 \%$ & $87 \%$ & $93 \%$ \\
\hline $\begin{array}{l}\text { 13. Mes besoins et ceux de mes proches / } \\
\text { amis ont été pris en compte lors de la } \\
\text { planification de mon voyage à } \\
\text { destination de l'autre ville. }\end{array}$ & $81 \%$ & $83 \%$ & $70 \%$ & $71 \%$ & $76 \%$ \\
\hline
\end{tabular}


Malgré ses limites, cette étude constitue la première exploration systématique d'un programme canadien de réacheminement pour des personnes atteintes du cancer suivant une radiothérapie. Elle offre des aperçus intéressants aux gestionnaires de programmes de ce genre, ainsi qu'à d'autres qui pourraient être amenés à développer des projets semblables. Dans l'ensemble, une proportion relativement importante des personnes interrogées a exprimé un avis favorable sur les soins. Cependant, des améliorations pourraient être apportées dans quelques domaines précis.
La diminution à terme de la satisfaction concernant la coordination a pu être influencée par deux facteurs. Le programme de réacheminement a été considérablement élargi durant les mois d'automne (septembre-décembre) et d'hiver (janvier-mars), deux périodes où le nombre de patients désireux d'y participer s'était accru de manière significative. Du personnel supplémentaire avait dû être engagé et affecté au programme, avant que le service n'ait pu être étendu. Dans l'intervalle, le personnel initial devait gérer un grand nombre de cas, et les temps

Tableau 3. Satisfaction des patients envers le Programme de réacheminement : questions portant sur le centre de traitement du cancer où la radiothérapie a été effectuée (échantillons trimestriels et globaux)

\begin{tabular}{|c|c|c|c|c|c|}
\hline Questions de la partie 2 & $\begin{array}{c}\text { Avr. -juin } 1999 \\
(\mathrm{n}=98)\end{array}$ & $\begin{array}{c}\text { Juil.-sept. } 1999 \\
(\mathrm{n}=121)\end{array}$ & $\begin{array}{l}\text { Oct.-déc. } 1999 \\
(n=164)\end{array}$ & $\begin{array}{c}\text { Jan.-mars } 2000 \\
\qquad(\mathrm{n}=83)\end{array}$ & $\begin{array}{c}\text { Total } \\
(n=466)\end{array}$ \\
\hline \multicolumn{6}{|c|}{$\begin{array}{l}\text { Proportion de répondants de chaque groupe } \\
\text { qui étaient d'accord avec l'énoncé }\end{array}$} \\
\hline $\begin{array}{l}\text { 14. Avant ma première visite, j'ai reçu } \\
\text { une information opportune et utile } \\
\text { sur le centre de traitement du cancer } \\
\text { où j'allais recevoir ma radiothérapie. }\end{array}$ & $81 \%$ & $85 \%$ & $79 \%$ & $72 \%$ & $79 \%$ \\
\hline $\begin{array}{l}\text { 15. Le processus d'inscription au centre } \\
\text { de traitement du cancer était simple. }\end{array}$ & $99 \%$ & $98 \%$ & $97 \%$ & $99 \%$ & $98 \%$ \\
\hline $\begin{array}{l}\text { 16. Le personnel du centre de traitement } \\
\text { du cancer où j'ai reçu ma } \\
\text { radiothérapie était courtois } \\
\text { et respectueux. }\end{array}$ & $100 \%$ & $100 \%$ & $100 \%$ & $100 \%$ & $100 \%$ \\
\hline $\begin{array}{l}\text { 17. J'étais satisfait(e) de la quantité } \\
\text { d'information que l'on m'a fournie } \\
\text { sur ma radiothérapie. }\end{array}$ & $91 \%$ & $96 \%$ & $94 \%$ & $98 \%$ & $94 \%$ \\
\hline $\begin{array}{l}\text { 18. On m'a informé(e) des services dont } \\
\text { je pourrais avoir besoin ou je voudrais } \\
\text { me prévaloir tels que diététistes } \\
\text { cliniques, travailleurs sociaux, } \\
\text { pharmaciens, aumôniers, } \\
\text { psychologues ou groupes de soutien. }\end{array}$ & $67 \%$ & $71 \%$ & $68 \%$ & $76 \%$ & $71 \%$ \\
\hline $\begin{array}{l}\text { 19. J'ai reçu d'excellents soins au centre } \\
\text { de cancérologie où j'ai suivi } \\
\text { ma radiothérapie. }\end{array}$ & $99 \%$ & $100 \%$ & $99 \%$ & $98 \%$ & $99 \%$ \\
\hline $\begin{array}{l}\text { 20. Mes conditions d'hébergement } \\
\text { étaient excellentes. }\end{array}$ & $95 \%$ & $86 \%$ & $90 \%$ & $98 \%$ & $91 \%$ \\
\hline $\begin{array}{l}\text { 21. Il m'était facile de me rendre de mon } \\
\text { lieu d'hébergement au centre } \\
\text { de traitement. }\end{array}$ & $100 \%$ & $97 \%$ & $99 \%$ & $96 \%$ & $98 \%$ \\
\hline $\begin{array}{l}\text { 22. J'ai reçu beaucoup de soutien de la } \\
\text { part des professionnels de la santé. }\end{array}$ & $95 \%$ & $92 \%$ & $94 \%$ & $91 \%$ & $92 \%$ \\
\hline $\begin{array}{l}\text { 23. Le personnel était sensible au fait que } \\
\text { j'étais à l'extérieur de ma région de } \\
\text { résidence et que je pouvais avoir des } \\
\text { besoins particuliers. }\end{array}$ & $88 \%$ & $91 \%$ & $91 \%$ & $91 \%$ & $91 \%$ \\
\hline $\begin{array}{l}\text { 24. Le fait de suivre mon traitement } \\
\text { dans une autre ville n'a entraîné, } \\
\text { ni pour moi ni pour ma famille, } \\
\text { un fardeau financier supplémentaire. }\end{array}$ & $64 \%$ & $58 \%$ & $56 \%$ & $57 \%$ & $56 \%$ \\
\hline $\begin{array}{l}\text { 25. En vertu de mon expérience, je } \\
\text { recommanderais le centre de } \\
\text { cancérologie où j'ai reçu ma } \\
\text { radiothérapie à d'autres membres } \\
\text { de ma famille ou à des amis s'ils } \\
\text { avaient besoin d'un tel traitement. }\end{array}$ & $98 \%$ & $98 \%$ & $95 \%$ & $100 \%$ & $96 \%$ \\
\hline
\end{tabular}


de réponse s'en ressentaient. En outre, durant les mois d'hiver (janvier-mars), les déplacements au Canada sont rendus difficiles par la neige et le verglas. Les activités de plein air sont réduites, et les gens sont confinés à l'intérieur. Une telle situation a pu renforcer le sentiment d'ennui que les patients disent avoir éprouvé, alors qu'ils suivaient une radiothérapie hors de leur région (Fitch et al., 2003).

Avant le traitement, de nombreux patients estimaient qu'ils n'avaient pas vraiment accès au personnel et au soutien du centre de cancérologie de leur région. C'est compréhensible, étant donné que la plupart de ces patients n'avaient pas eu de rendez-vous dans ces établissements et qu'ils ne connaissaient pas les oncologues ni d'autres soignants. Ceux qui avaient eu un rendezvous n'auraient vraisemblablement eu qu'un entretien sur la nécessité d'être traités ailleurs. Il est fort probable qu'à moins d'avoir été explicitement informés qu'ils pouvaient bénéficier des services de soutien du centre de cancérologie de leur région, les patients aient eu l'impression qu'ils ne pouvaient pas en utiliser les services.

Cet intervalle de prétraitement délimite le moment où le diagnostic a été annoncé et où l'individu attend le début du traitement. C'est souvent une période de grande anxiété pour les patients, qui craignent alors que le cancer ne croisse pendant qu'ils attendent (Fitch et al., 2003). C'est aussi un moment où les patients ne sont pas nécessairement informés des services qui sont offerts pour leur venir en aide. Souvent, ils n'apprennent l'existence de ces services que lorsqu'ils commencent à fréquenter le centre de traitement (Degrasse et Hugo, 1996). Des améliorations pourraient donc être apportées à la prestation d'information sur les services à ces patients, ce qui faciliterait l'accès aux services de soutien durant l'intervalle de prétraitement.

La prestation d'information sur le cancer, la radiothérapie et le centre de cancérologie vers lequel les patients sont réorientés, a également été jugée insuffisante. Le problème du manque d'information se posait déjà pour ces patients avant le début de leur traitement. Mais une fois arrivés au centre de cancérologie de réacheminement, leur traitement commencé, ils se sont estimés satisfaits de l'information qu'ils recevaient. Cette observation soulève des questions sur la nature des conversations qui s'engagent (ou non) avec les patients avant qu'ils ne partent pour leur traitement. Le personnel des centres locaux doit être sensible aux besoins d'information des patients qui s'inscrivent au programme de réacheminement. De même, le programme de réacheminement devrait revoir le type d'information qu'il présente et les dates choisies afin de transmettre cette information aux patients. Cependant, compte tenu que ces problèmes ont également été évoqués à propos des modalités d'inscription au centre de cancérologie local et du temps d'écoute disponible, on pourrait être amené à poser la question suivante, à savoir quelle responsabilité le personnel du centre de cancérologie local pense devoir assumer à l'égard de ces patients. Il semblerait qu'il considère qu'il a peu de responsabilité envers ces derniers, ou bien les observations reflètent la lourde charge de travail et la forte fréquentation des centres locaux.

La prestation d'information sur les services de soins de soutien était davantage perçue comme un problème par les personnes qui étaient soignées aux États-Unis. Dans les établissements canadiens, des mécanismes particuliers avaient été mis au point pour résoudre ce problème. Par exemple, au centre de Thunder Bay, une session d'information hebdomadaire animée conjointement par une travailleuse sociale et une radiothérapeute, était proposée aux patients. Étant donné que la majorité des patients réorientés vers des établissements canadiens étaient allés à Thunder Bay, cette session d'information a pu faire la différence entre les centres canadiens et américains. Il semblerait important pour tous les centres accueillant des patients venus se faire soigner de l'extérieur d'organiser régulièrement des sessions d'information.

Un nombre moins important de femmes que d'hommes avaient jugé satisfaisants certains aspects des soins relatifs aux dispositions de voyage et aux services disponibles. Les tâches et les responsabilités qui incombent à une femme influencent sans nul doute le degré de difficulté qu'elle pourra éprouver à rester absente du foyer pendant un certain temps. De nombreuses femmes ressentent le besoin de prendre des dispositions vis-à-vis des responsabilités du ménage, de la famille et de leur travail pour la durée du traitement. Bien que malades, les femmes estiment qu'il leur incombe encore de s'occuper des enfants et des travaux ménagers (Fitch, Bunston et Elliot, 1999) et elles y renoncent difficilement. Pour nombre d'entre elles, ces dispositions sont une source de stress supplémentaire, à un moment où elles se sentent déjà vulnérables. Les femmes qui quittent leur région pour se faire soigner d'un cancer ont décrit leur crainte d'imposer un fardeau de plus à leur famille, avec les changements de responsabilités à la maison et la nécessité de se faire conduire (Fitch et al., 2003). Des femmes australiennes vivant en milieu rural, contraintes de se déplacer à la suite d'un diagnostic de cancer du sein, ont fait état des mêmes préoccupations (McGrath et al., 1999a, 1999b).

Tableau 4. Satisfaction envers certains aspects du Programme de réacheminement, par sexe

\begin{tabular}{|c|c|c|c|}
\hline \multirow[b]{2}{*}{$\begin{array}{l}\text { Questions où il y avait une } \\
\text { différence significative }\end{array}$} & \multicolumn{3}{|c|}{$\begin{array}{l}\text { Proportion de répondants dans chaque } \\
\text { groupe qui étaient d'accord }\end{array}$} \\
\hline & Femmes & Hommes & $\begin{array}{l}\text { Signification } \\
\text { statistique }\end{array}$ \\
\hline $\begin{array}{l}\text { Il était clair pour moi que la décision de } \\
\text { voyager vers une autre ville pour recevoir } \\
\text { ma radiothérapie me revenait à moi } \\
\text { seul(e) et que j'avais le droit de refuser. }\end{array}$ & $86 \%$ & $96 \%$ & $\begin{array}{l}\mathrm{DDL}=1 \\
\chi^{2}=6,08 \\
\mathrm{p}=0,025\end{array}$ \\
\hline $\begin{array}{l}\text { J'ai reçu tous les renseignements dont } \\
\text { j'avais besoin sur les démarches } \\
\text { nécessaires à l'obtention de ma } \\
\text { radiothérapie dans une autre ville. }\end{array}$ & $71 \%$ & $88 \%$ & $\begin{array}{l}\mathrm{DDL}=1 \\
\chi^{2}=5,94 \\
\mathrm{p}=0,025\end{array}$ \\
\hline $\begin{array}{l}\text { Mes besoins et ceux de mes proches / } \\
\text { amis ont été pris en compte lors de la } \\
\text { planification de mon voyage à } \\
\text { destination de l'autre ville. }\end{array}$ & $72 \%$ & $84 \%$ & $\begin{array}{l}\mathrm{DDL}=1 \\
\chi^{2}=4,2 \\
\mathrm{p}=0,05\end{array}$ \\
\hline $\begin{array}{l}\text { On m'a informé(e) des services dont je } \\
\text { pourrais avoir besoin ou je voudrais me } \\
\text { prévaloir tels que diététistes cliniques, } \\
\text { travailleurs sociaux, pharmaciens, } \\
\text { aumôniers, psychologues ou groupes } \\
\text { de soutien. }\end{array}$ & $65 \%$ & $78 \%$ & $\begin{array}{l}\mathrm{DDL}=1 \\
\chi^{2}=4,14 \\
\mathrm{p}=0,05\end{array}$ \\
\hline
\end{tabular}


Les variations dans les observations selon le sexe peuvent également refléter le fait que les hommes et les femmes n'ont pas les mêmes mécanismes d'adaptation. Des travaux récents sur les expériences de groupes d'entraide (Gray, Fitch, Davis et Phillips, 1997a, 1997b) ont révélé des différences intéressantes entre les hommes et les femmes. Pour la plupart, les femmes attendent un soutien de leur famille et de leurs amis. Elles cherchent l'occasion de pouvoir exprimer leurs sentiments et discuter d'expériences partagées. C'est ce à quoi elles consacrent le plus de temps dans les groupes d'entraide avec d'autres patients. Les hommes, quant à eux, n'ont pas l'habitude de s'entourer de confidents intimes (Gray, Fitch, Phillips, Labrecque et Fergus, 2000). Ceux qui vivent en couple peuvent très bien ne parler qu'à leur épouse ou partenaire de ce qui leur arrive. Dans les groupes d'entraide, ils ont tendance à privilégier l'obtention d'informations concrètes. Étant données ces différences, les femmes peuvent éprouver plus de difficulté à s'éloigner de leur maison et de leur réseau de soutien pour un traitement. On pourrait également en déduire qu'un certain nombre d'obligations sociales élargies devraient être clarifiées avant le départ. Le fait que les femmes aient ou non le sentiment de pouvoir négocier librement avec le personnel du programme de réacheminement peut influencer leur perception relativement au degré de prise en compte de leurs besoins lors de l'organisation des dispositions de voyage.

Les préoccupations sur les aspects financiers doivent aussi retenir notre attention. Lorsqu'il existe un processus de remboursement, les patients doivent en être tenus informés, et bien comprendre comment il fonctionne et quelles sont exactement les dépenses qui sont couvertes. Une évaluation sur la capacité des personnes à s'acquitter des frais et à attendre un remboursement ultérieur est nécessaire. Certains estiment en effet que devoir payer immédiatement les services occasionne une gêne financière difficile à surmonter.

Finalement, les patients qui ont été traités dans un centre de cancérologie à l'extérieur de leur région indiquent un manque de confiance dans les soins de suivi dont ils pourraient bénéficier au

\section{Références}

Abbey-Livingstone, D. \& Associates. (1990). The needs of Ontario cancer patients: An assessment 1990. Toronto: Canadian Cancer Society.

Carroll-Johnson, R.M., Gorman, L.M., \& Bush, M.J. (1998). Psychosocial nursing care along the cancer continuum. Pittsburgh, PA: Oncology Nursing Press.

Davis, C., \& Girgis, A. (1998). Needs assessment of rural and remote women travelling to the city for breast cancer treatment. Australia and New Zealand Journal of Public Health, 22(5), 525-527.

Degrasse, C., \& Hugo, K. (1996). Supportive care needs of women undergoing breast diagnostics and their families: A focus on nursing interventions. Canadian Oncology Nursing Journal, 6(4), 185-190.

Fitch, M.I., Bunston, T., \& Elliot, M. (1999). When Mom's sick: Changes in a mother's role and in the family after her diagnosis of cancer. Cancer Nursing, 22(1), 58-63.

Fitch, M.I., Gray, R.E., McGowan, T., Brunskill, I., Steggles, S., Sellick, S. et al., (2003). Travelling for radiation cancer treatment: Patient perspectives. Psycho-Oncology, 12, 664694.

Given, B.A., Given, C.W., \& Harlan, A.N. (1994). Strategies to meet the needs of the rural poor. Seminars in Oncology Nursing, 10(2), 114-122. retour. Durant le traitement, lorsqu'un patient voit chaque jour les membres d'une équipe soignante, un climat de confiance finit par s'installer. S'adresser pour des soins de suivi à un professionnel de la santé étranger à cette expérience revient à aller voir quelqu'un que le patient ne connaît pas et avec qui il n'a pas eu l'opportunité d'établir une relation de confiance. En outre, si les étapes du processus des soins de suivi ne sont pas claires, les patients pourraient entretenir des doutes sur la manière dont ces soins seront gérés. Il convient donc de fournir au patient une information explicite sur le processus des soins de suivi, y compris les services à appeler en cas d'effets secondaires indésirables du traitement.

De même, la première visite au centre de cancérologie local doit être plus longue qu'un rendez-vous de suivi ordinaire, de manière à instaurer un dialogue avec le patient à propos de son expérience de soins dans un autre centre et à favoriser l'établissement d'une relation de confiance.

\section{Répercussions pour les infirmières en oncologie}

Il y a plusieurs répercussions pour les infirmières chargées de soigner les patients qui doivent se déplacer pour une radiothérapie. Avant de partir, les patients tireraient un grand profit de la prestation d'informations sur les dispositions de voyage, l'appui financier, les programmes de soutien, la région de destination et le centre de cancérologie qui va les accueillir. Le fait d'être au courant des programmes de soutien existants dans leur propre collectivité et de savoir à qui ils peuvent demander de l'aide, pourrait s'avérer utile pour les patients pendant et après leur série de traitement. Les infirmières doivent être bien conscientes que les patients qui retournent dans le centre de cancérologie de leur région, après un traitement à l'extérieur, auront des questions concernant leurs soins de suivi et risquent de manquer de confiance dans l'équipe soignante. Des efforts sont nécessaires pour établir une relation de confiance avec ces personnes.

Gray, R.E., Fitch, M., Davis, C., \& Phillips, C. (1997b). Interviews with men about their self-help experiences. Journal of Palliative Care, 13(1), 15-21.

Gray, R.E., Fitch, M., Davis, C., \& Phillips, C. (1997a). A qualitative study of breast cancer self-help groups. Journal of Psychosocial Oncology, 6, 279-289.

Gray, R.E., Fitch, M., Phillips, C., Labrecque, M., \& Fergus, K. (2000). Managing the impact of illness: The experiences of men with prostate cancer and their spouses. Journal of Health Psychology, 5(4), 531-548.

McGrath, P., Patterson, C., Yates, P., Treloars, S., Oldenburg, B., \& Loos, C. (1999a). A study of post-diagnosis breast cancer concerns for women living in rural and remote Queensland (Part I): Personal concerns. Australian Journal of Rural Health, 7, 34-42.

McGrath, P., Patterson, C., Yates, P., Treloars, S., Oldenburg, B., \& Loos, C. (1999b). A study of post-diagnosis breast cancer concerns for women living in rural and remote Queensland (Part II): Support issues. Australian Journal of Rural Health, 7, 43-52.

Société canadienne du cancer (1992). Rapport final sur les besoins des personnes atteintes de cancer au Canada. Toronto : Auteur. 